\title{
Una demostración simple de la desigualdad de Bell basada en la teoría elemental de conjuntos
}

\author{
A simple proof of Bell's inequality based on elementary set theory
}

\author{
Jorge Pinochet, Daniel Rojas-Líbano
}

Facultad de Educación, Universidad Alberto Hurtado, Santiago Centro, Santiago, Chile

Recebido em 28 de janeiro de 2016. Revisado em 22 de março de 2016. Aceito em 28 de março de 2016

La desigualdad de Bell ha sido considerada uno de los resultados más profundos de la ciencia. Debido a su importancia, se han propuesto diversos trabajos que han intentado hacerla accesible a los no especialistas. En este artículo se presenta una nueva demostración de la desigualdad de Bell, basada en la teoría elemental de conjuntos, y que destaca por su simplicidad matemática. La exposición está destinada principalmente a estudiantes no graduados de física, y pone especial énfasis en clarificar el significado y los alcances del teorema de Bell en el contexto de la paradoja de Einstein-Podolski-Rosen-Bohm.

Palabras clave: Desigualdad de Bell, paradoja EPRB, desigualdad de Wigner-d'Espagnat.

Bell's inequality has been considered one of deepest results of science. Due to its importance, several works have attempted to make it accessible to non-specialists. In this article we present a new proof of Bell's inequality based on elementary set theory, which stands out for its mathematical simplicity. The exposition is mainly intended for both graduate and undergraduate physics students. The article places particular emphasis on clarifying the meaning and implications of Bell's theorem in the context of the Einstein-Podolsky-Rosen-Bohm paradox.

Keywords: Bell's inequality, EPRB paradox, Wigner-d'Espagnat's inequality.

\section{Introducción}

La primera mitad del siglo XX fue testigo de un debate sobre los fundamentos de la Mecánica Cuántica (MC) que enfrentó a dos gigantes de la física: Albert Einstein y Neils Bohr. El punto culminante del debate lo constituye un experimento imaginario ideado por Einstein en colaboración con Boris Podolski y Nathan Rosen [1]. En honor de sus autores, el experimento ha llegado a conocerse con el nombre de argumento EPR, experimento EPR o paradoja $E P R$. Más tarde, David Bohm reformuló y simplificó el experimento imaginario, razonando sobre el caso de dos protones que se encuentran en un estado cuántico apropiado [2]. En concreto, Einstein y sus colaboradores introducen un ingenioso mecanismo que permite eludir las reglas cuánticas, asociando a los protones más variables de las que autoriza la MC. $\mathrm{El}$ argumento EPR concluye entonces que la MC

*Endereço de correspondência: japinochet@gmail.com es incompleta o aproximada, porque la descripción suministrada por esta teoría no contiene todas las variables en juego. Según Einstein, estas variables no especificadas serían las responsables de las características probabilísticas de la MC. El argumento de Einstein, Podolski, Rosen y Bohm (EPRB) intenta probar que la MC es incompleta o aproximada. El argumento EPRB se basa en un supuesto denominado principio de localidad de Einstein, según el cual dos objetos separados una distancia arbitrariamente grande solo pueden interactuar físicamente mediante influencias cuya rapidez de propagación sea inferior a la de la luz [3].

La respuesta de Bohr apareció pocos meses después de la publicación del argumento EPR [4]. En lo fundamental, Borh no cuestiona este argumento, sino que se limita a objetar los supuestos en que se basa. Hasta el año 1964, estar de acuerdo con Einstein, o por el contrario, estar de acuerdo con Bohr sobre las conclusiones del argumento EPRB 
era básicamente una cuestión de concepciones filosóficas. Sin embargo, en el año 1964, John S. Bell publicó un teorema que constituye una extensión del argumento EPRB[5], y que permitió sacar el debate del plano filosófico para situarlo en el terreno experimental, al menos en principio. El teorema de Bell o desigualdad de Bell constituye una prueba de imposibilidad que adopta la forma de una desigualdad matemática. En lo fundamental, el teorema afirma que el argumento EPRB es incompatible con las predicciones estadísticas de la MC $[5,6]$. Desde la publicación de la desigualdad de Bell se han invertido grandes esfuerzos en clarificarla, y se han realizado diversos experimentos que han intentando contrastarla con las predicciones de la MC. Dentro de los límites experimentales, los resultados respaldan las predicciones cuánticas, revelando una clara violación de la desigualdad de Bell [7-10].

Dada su importancia, la desigualdad de Bell ha sido calificada como el descubrimiento más profundo de la ciencia [11]. De ahí que se hayan publicado diversos trabajos que han intentado simplificar y divulgar el aporte pionero de Bell [6, 12-25]. De todos los trabajos publicados hasta ahora, el que mejor parece conjugar simplicidad y profundidad es un artículo debido a d'Espagnat [3], donde se introduce una versión elemental del teorema de Bell conocida como desigualdad de Wigner-d'Espagnat $(W D)[6,12]$.

El presente trabajo tiene un doble propósito. El primero es clarificar el significado y los alcances del teorema de Bell en el contexto del argumento EPRB. El segundo es proporcionar una nueva demostración de la desigualdad WD, que destaca por su simplicidad matemática. La demostración sólo utiliza conceptos y operaciones de la teoría elemental de conjuntos, así como algunas nociones básicas de probabilidad. Un aspecto clave de la exposición es el uso de una sencilla notación que evidencia la continuidad de ideas entre el argumento EPRB y la desigualdad de Bell, y que permite que esta última surja naturalmente a partir de la teoría de conjuntos.

Para alcanzar los objetivos propuestos, el artículo comienza presentado una versión simplificada y no técnica del argumento EPRB. A continuación se introduce la demostración de la desigualdad WD basada en la teoría elemental de conjuntos, presentándola como una extensión directa del argumento EPRB. Finalmente se contrasta la desigualdad WD con las predicciones probabilísticas de la $\mathrm{MC}$, tanto en términos numéricos como gráficos, poniendo énfasis, nuevamente, en su relación con el argumento EPRB.

\section{Eludiendo las reglas cuánticas: El argumento EPRB}

Considérese un sistema físico compuesto por dos protones en un estado cuántico denominado singlete. La importancia de este estado para el desarrollo del argumento EPRB quedará en evidencia más adelante. Luego de ser emitidos desde una fuente, los protones se separan viajando en sentidos opuestos a lo largo de un mismo eje, sin sufrir ninguna perturbación. Supóngase que cada protón se dirige hacia un dispositivo especializado en medir componentes de spin. Denotemos como $I$ al dispositivo que se encuentra a la izquierda de la fuente, y como $D$ al que se encuentra a la derecha, donde las distancias de $I$ y $D$ a la fuente son iguales. Para el problema que nos ocupa no es necesario ahondar en los aspectos técnicos de una componente de spin; lo único que es preciso tener presente es que dicha componente es una propiedad de las partículas que se mide respecto de una orientación o eje determinado, y que una vez escogido dicho eje, esta propiedad solo pueden tomar dos valores denotados convencionalmente como $(+) \mathrm{y}(-)$.

Para denotar la componente de spin sobre un eje determinado, se usará un superíndice izquierdo para el protón que viaja hacia $I$, y un superíndice derecho para el protón que viaja hacia $D$. Por ejemplo, si la componente del protón que viaja hacia $I$ tiene el valor $(+)$ sobre el eje $x$ se usará la notación ${ }^{+} x$. En forma análoga, si la componente del protón que viaja hacia $D$ tiene el valor $(-)$ sobre el eje $y$ se usará la notación $y^{-}$. Es importante tener presente que las reglas de la MC solo permiten medir una componente de spin para cada protón, y que dichas reglas son consistentes con las resultados experimentales. Como se verá enseguida, son precisamente estas reglas y resultados experimentales los que el argumento EPRB intenta eludir.

La importancia del estado singlete para el desarrollo del argumento EPRB reside en la siguiente propiedad fundamental: cuando $I$ y $D$ están orientados sobre un mismo eje, la MC predice resultados opuestos para las medidas conjuntas de spin sobre dos protones en estado singlete. Por lo tanto, si se supone que tanto $I$ como $D$ están orientados sobre el 
eje $x$, entonces toda medida ${ }^{+} x$ permite predecir con certeza una medida $x^{-}$, y viceversa. De igual modo, toda medida ${ }^{-} x$ permite predecir con certeza una medida $x^{+}$, y viceversa. La misma lógica se aplica al eje $y$ o a cualquier otro eje de medición. Desde la perspectiva del argumento EPRB la situación que interesa analizar es cuando las orientaciones relativas de $I$ y $D$ son perpendiculares, tal como muestra la Fig. 1, donde $I$ está orientado sobre el eje $x$ y $D$ sobre el eje $y$. En estas condiciones, a partir de las propiedades del estado singlete se sigue que si $I$ mide ${ }^{+} x$, entonces podemos inferir con certeza que si $D$ estuviera orientado sobre el eje $x$, el resultado de su medición sería $x^{-}$. Como se trata de una medida conjunta de spin, al mismo tiempo que $I$ mide ${ }^{+} x$, podemos imaginar que $D$ mide $y^{+}$, lo que permite inferir con certeza que si Iestuviera orientado sobre el eje $y$, el resultado de su medición sería $^{-} y$. Por lo tanto, al protón de la izquierda le asignamos una componente de spin medida ${ }^{+} x, \mathrm{y}$ al protón de la derecha le asignamos una componente inferida $x^{-}$, lo que puede escribirse como ${ }^{+} x^{-}$. Del mismo modo, al protón de la derecha le asignamos una componente medida $y^{+}$, y el protón de la izquierda le asignamos una componente inferida ${ }^{-} y$, lo que puede escribirse como ${ }^{-} y^{+}$. Usando notación de pares ordenados, estas dos conclusiones del argumento EPRB pueden resumirse como $\left({ }^{+} x^{-},{ }^{-} y^{+}\right)$. No obstante, esta conclusión contradice las reglas de la MC y los resultados experimentales, ya que según estas reglas y resultados es imposible asignar simultáneamente dos componentes de spin a un mismo protón sobre dos ejes distintos como $x$ e $y$. En concreto, considerando que $I$ midió ${ }^{+} x$ y $D$ midió $y^{+}$, el único resultado compatible con la MC y con los experimentos es $\left({ }^{+} x, y^{+}\right)$. Vemos entonces que el argumento EPRB ha permitido eludir las reglas cuánticas, asociando a los protones más componentes de spin de las permitidas.
Es el momento de introducir el criterio de realidad de EPRB [1], que puede parafrasearse del siguiente modo: Si es posible inferir con certeza el resultado de la medición de una propiedad física, entonces dicha propiedad tiene una existencia real. Se aprecia que este criterio busca independizar el concepto de realidad física del proceso de medición u observación (esta es la tesis central del realismo filosófico). Ahora bien, como el resultado $\left({ }^{+} x^{-},{ }^{-} y^{+}\right)$incorpora más propiedades físicas (reales) que el resultado cuántico $\left({ }^{+} x, y^{+}\right)$, y dado que según el argumento EPRB una teoría no está completa hasta que cada propiedad física tenga su contraparte en la teoría [1], se concluye que la $\mathrm{MC}$ es incompleta o aproximada. Se puede arribar a esta misma conclusión intercambiando los ejes de medición asignados a $I$ y $D$, así como las componentes de spin para cada eje, de modo que el resultado obtenido es completamente general.

Es muy importante tener presente que toda la argumentación EPRB depende del principio de localidad de Einstein, según el cual dos objetos separados una distancia arbitrariamente grande no pueden interactuar físicamente mediante influencias cuya rapidez de propagación sea superior a la de la luz. Por lo tanto, si se acepta el principio de localidad, se concluye que no puede existir ningún tipo de interacción que implique que una medida efectuada sobre un protón pueda afectar o perturbar de algún modo al otro protón, ya que al momento de realizar las medidas conjuntas de spin, la distancia entre $I$ y $D$ puede hacerse tan grande como se desee. Se aprecia entonces que si se rechaza el principio de localidad, toda la argumentación EPRB queda invalidada, pues resulta imposible considerar a los protones como entidades independientes o separadas. Por ejemplo, dados dos protones $A$ y $B$ en estado singlete, si suponemos que una medida ${ }^{+} x$ efectuada sobre $A$ puede afectar a $B$, entonces ya no es posible predecir con certeza que la componente de $B$ es

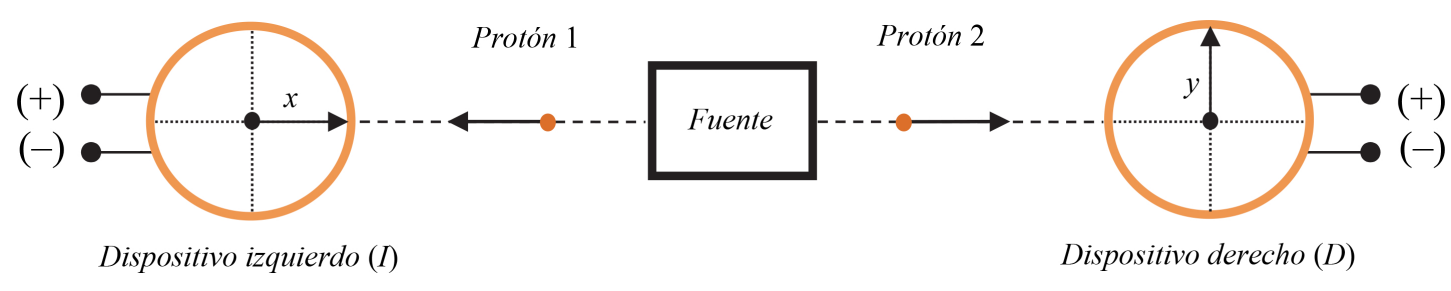

Figura 1: Dos protones en estado singlete viajan a lo largo de un mismo eje en sentidos opuestos hacia dos dispositivos de medición: $I$ (izquierda), orientado sobre el eje $x$, y $D$ (derecha), orientado sobre el eje $y$. Solo existen dos posibles valores para la medición de spin de cada dispositivo: $(+)$ y $(-)$. Cada vez que $/$ obtiene el resultado $(+/-), D$ obtiene $(-/+)$. 
$x^{-}$puesto que la medida sobre $A$ ha generado una perturbación que modifica el spin de $B$.

El razonamiento EPRB nos enfrena a una disyuntiva que algunos han considerado una paradoja: $\mathrm{O}$ bien se renuncia al principio de localidad, en cuyo caso es posible mantener la completitud de la MC, o bien se renuncia a la completitud, en cuyo caso es posible preservar el principio de localidad. Dado que el abandono de este último conlleva un conflicto con la teoría de la relatividad de Einstein, según la cual ninguna interacción puede propagarse más rápidamente que la luz en el vacío, se entiende que desde la perspectiva del argumento EPRB sea inevitable concluir que la MC es aproximada o incompleta. Para Einstein, las propiedades físicas de una partícula, tales como el spin, son descritas por la MC en términos probabilísticos debido a la existencia de variables no especificadas. Desde este punto de vista, la aleatoriedad no constituiría un rasgo fundamental de la realidad física; al contrario, si fuese posible tener en cuenta las variables no especificadas, las propiedades de una partícula podrían determinarse con precisión.

El hallazgo fundamental realizado por Bell es que el argumento EPRB conduce a resultados experimentales incompatibles con las predicciones estadísticas de la MC. Para llegar a esta conclusión, Bell se percató que el argumento EPRB solo funciona cuando $I$ y $D$ se encuentran orientados sobre ejes perpendiculares, como $x$ e $y$. Sin embargo, si se considera una situación más general, el panorama cambia dramáticamente. En efecto, imaginemos que $I$ y $D$ pueden efectuar medidas conjuntas de spin sobre tres ejes arbitrarios $a, b$ y $c$ que no forman necesariamente ángulos rectos entre sí, y donde cada dispositivo pueda realizar un gran número de medidas conjuntas sobre un conjunto amplio de pares de protones en estado singlete. Bajo estas condiciones se puede probar que se satisface la siguiente desigualdad, que resulta violada para algunas orientaciones de $a, b$ y $c$ :

$$
n u m\left({ }^{+} a, b^{+}\right) \leqslant n u m\left({ }^{+} a, c^{+}\right)+\operatorname{num}\left({ }^{+} c, b^{+}\right)
$$

Esta es la forma más simple del teorema de Bell, denominada desigualdad WD, y que se sigue directamente del argumento EPRB. Cada término de esta desigualdad denota el número (num) de mediciones conjuntas de spin con los resultados que aparecen indicados. El objetivo de la siguiente sec- ción es demostrar la desigualdad WD en base a la teoría elemental de conjuntos.

\section{El argumento EPRB conduce a una desigualdad}

Para llevar a cabo la demostración de la desigualdad WD, consideremos los mismos dispositivos de medición $I$ y $D$ introducidos antes. A diferencia de la sección anterior, donde interesaba medir las componentes de spin sobre un solo par de protones en estado singlete, ahora interesa efectuar un conjunto amplio de medidas conjuntas sobre un gran número de pares de protones en estado singlete. Para ello, supóngase que la fuente emite secuencialmente cada par de protones, y que cada dispositivo puede ajustarse para medir el spin sobre tres ejes arbitrarios $a$, $b$ y $c$, que no forman necesariamente ángulos rectos entre sí. Como antes, las componentes de spin para cada eje de medición solo pueden tomar los valores $(+) \mathrm{y}(-)$.

Aunque en la práctica resulta imposible medir el spin sobre los ejes $a, b$ y csimultáneamente, si se aceptan las reglas del argumento EPRB, es posible asociar componentes de spin simultáneas a cada protón sobre los tres ejes. Como ejemplo, imaginemos que $I$ se encuentra orientado sobre el eje $a$ y $D$ sobre el eje $b$; si una medida conjunta sobre un par de protones produce el resultado $\left({ }^{+} a, b^{+}\right)$, entonces no solo podemos inferir el resultado $\left({ }^{+} a^{-},{ }^{-} b^{+}\right)$, sino que también podemos suponer la existencia de componentes de spin sobre el eje $c$, de modo que necesariamente el par de protones tendrá o bien componentes $\left({ }^{+} a^{-},{ }^{-} b^{+},{ }^{+} c^{-}\right)$o bien componentes $\left({ }^{+} a^{-}\right.$, $\left.{ }^{-} b^{+},{ }^{-} c^{+}\right)$. Siguiendo con esta línea de razonamiento, supongamos que una medida conjunta produce el resultado $\left({ }^{+} a, c^{+}\right)$. Así, podemos inferir el resultado $\left({ }^{+} a^{-},{ }^{-} c^{+}\right)$, lo que permite concluir que el par de protones tendrá necesariamente o bien componentes $\left({ }^{+} a^{-},{ }^{-} b^{+},{ }^{-} c^{+}\right)$o bien componentes $\left({ }^{+} a^{-},{ }^{+} b^{-}\right.$, $\left.{ }^{-} c^{+}\right)$. Lo mismo se aplica al resultado de cualquier otra medida conjunta. Se observa que esta notación no permite distinguir entre mediciones e inferencias, lo que es consistente con el argumento EPRB. Por lo tanto, en el contexto del argumento EPRB es posible asociar componentes de spin a cada protón sobre los tres ejes de medición, tal como ilustra la Fig. 2.

Las consideraciones anteriores exigen introducir una sutil e importante ampliación del significado 


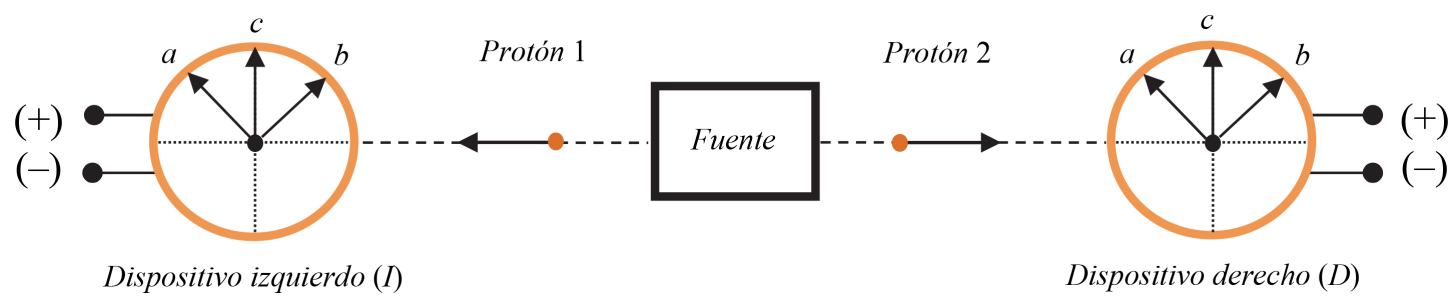

Figura 2: Dos protones en estado singlete viajan a largo de un mismo eje en sentidos opuestos hacia dos dispositivos de medición $I$ y $D$, cada uno de los cuales puede medir las componentes de spin sobre tres ejes arbitrarios, $a, b$ y $c$. Solo existen dos posibles resultados para las medidas de spin de $I$ y $D:(+)$ y $(-)$. Cada vez que el resultado de una medida efectuada por $I$ es $(+/-)$, el resultado de une medida efectuada por $D$ es $(-/+)$, y viceversa.

de una notación del tipo ${ }^{+} a^{-}$. A diferencia de la sección anterior, donde la notación para los ejes $x$ e $y$ permitía dar cuenta de medidas de spin para pares individuales de protones, la notación para los ejes $a$, $b$ y $c$ empleada en esta sección representará un gran número de medidas de spin. Por tanto, una notación del tipo ${ }^{+} a^{-}$designará un conjunto de medidas como el siguiente:

$$
{ }^{+} a^{-}={ }^{+} a_{1}^{-},{ }^{+} a_{2}^{-},{ }^{+} a_{3}^{-}, \ldots,{ }^{+} a_{m}^{-}
$$

donde los subíndices numéricos denotan las componentes de spin para cada medición efectuada, desde la 1 hasta la $m$. La misma lógica se aplica a los restantes ejes de medición para cada conjunto de componentes. Imaginemos entonces que la fuente comienza a emitir secuencialmente un gran número de pares de protones en estado singlete, y que para cada par registramos cuidadosamente los resultados de las medidas de las componentes de spin. Es posible organizar estos resultados en los ocho conjuntos que aparecen en la Fig. 3. En efecto, dado que existen tres ejes, cada uno de los cuales está asociado a dos valores, $(+)$ y $(-)$, se sigue que hay $2^{3}=8$ conjuntos de componentes, tal como muestra la figura.
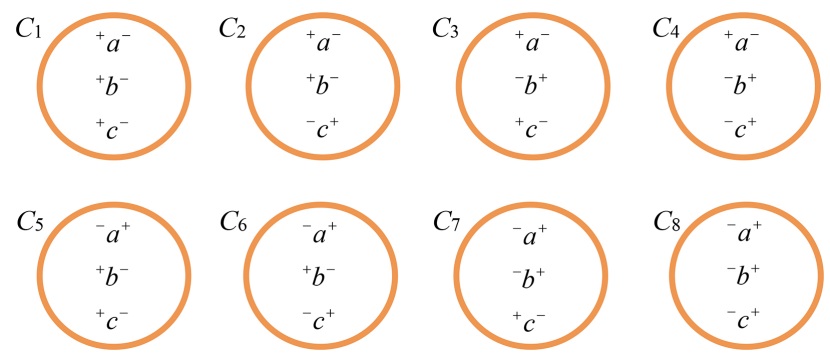

Figura 3: Los ocho conjuntos $\left(C_{1}, C_{2}, \ldots C_{8}\right)$ que agrupan todas las componentes de spin sobre los ejes $a, b$ y $c$. Para cada eje, la componente de spin solo puede tomar dos valores, denotados mediante los superíndices $(+)$ y $(-)$.
A continuación procederemos con nuestra demostración elemental de la desigualdad de Bell. Al aplicar la teoría de conjuntos es posible construir diversas relaciones matemáticas. Sin embargo, solo algunas de ellas son pertinentes para el problema que nos ocupa. Aquí nos interesa demostrar la Ec. (1) de la sección anterior. Aplicando las operaciones de unión e intersección a los conjuntos $C_{2}, C_{3}, C_{4} \mathrm{y}$ $C_{7}$ de la Fig. 3 se obtiene:

$$
\left(C_{3} \cap C_{4}\right) \subset\left[\left(C_{2} \cap C_{4}\right) \cup\left(C_{3} \cap C_{7}\right)\right]
$$

A partir de los conjuntos ilustrados en la Fig. 3 y definidos antes, la Ec. (2) es equivalente a:

$$
\left({ }^{+} a^{-},{ }^{-} b^{+}\right) \subset\left[\left({ }^{+} a^{-},{ }^{-} c^{+}\right) \cup\left({ }^{+} c^{-},{ }^{-} b^{+}\right)\right]
$$

Utilizando propiedades básicas de la teoría de conjuntos se sigue directamente que:

$$
\begin{array}{r}
\operatorname{num}\left({ }^{+} a^{-},{ }^{-} b^{+}\right) \leqslant \operatorname{num}\left({ }^{+} a^{-},{ }^{-} c^{+}\right)+ \\
\operatorname{num}\left({ }^{+} c^{-},{ }^{-} b^{+}\right)
\end{array}
$$

donde num denota la cardinalidad (número de elementos) de cada conjunto. Por ejemplo, el término de la izquierda representa el número de medidas conjuntas de spin con componentes ${ }^{+} a^{-} \mathrm{y}^{-} b^{+}$. Nótese que al intercambiar los ejes, así como las componentes para cada eje, es posible construir múltiples desigualdades análogas a la Ec. (4). Para que la Ec. (4) de cuenta de componentes de spin que en principio puedan determinarse experimentalmente, cada término de la desigualdad debe estar en función de una componente para cada eje, ya que esto es lo que exigen las reglas de la MC y los resultados experimentales. Para ello se requiere eliminar o bien los superíndices (+) o bien los superíndices (-). Optando por lo último: 


$$
\operatorname{num}\left({ }^{+} a, b^{+}\right) \leqslant \operatorname{num}\left({ }^{+} a, c^{+}\right)+\operatorname{num}\left({ }^{+} c, b^{+}\right)
$$

Esta es la forma más simple de la desigualdad WD, introducida en la sección anterior. Cabe insistir en que la Ec. (5) establece relaciones entre cantidades que en principio son empíricamente contrastables, ya que cada término es función de dos ejes de medición, uno para $I$ y otro para $D$, y cada eje tiene asociada una sola componente de spin. Además, cada término depende de dos ejes distintos, lo que resulta de gran importancia para el problema que nos ocupa, pues como quedará en evidencia en la última sección, las medidas conjuntas efectuadas sobre un mismo eje no generan conflicto entre el argumento EPRB y la MC. La Ec. (5) establece que el número de mediciones conjuntas con resultados ${ }^{+} a \mathrm{y} c^{+}$, sumada al número de mediciones con resultados ${ }^{+} c y b^{+}$, es siempre mayor o igual que el número de mediciones conjuntas con resultados ${ }^{+} a \mathrm{y}^{+}$.

Es importante enfatizar que, sin importar el número de medidas de spin con resultados ${ }^{+} a, b^{+} \mathrm{y}{ }^{+} c$, la Ec. (5) siempre es válida, lo cual puede verificarse fácilmente construyendo una tabla como la que aparece en la Fig. 4, donde cada columna representa un eje determinado, y donde cada fila representa una medida de spin sobre ese eje. Si el lector construye su propia tabla y la completa de forma arbitraria, observará que el número de filas ${ }^{+} a \mathrm{y}^{+}$sumada al número de filas $b^{+} \mathrm{y}{ }^{+} c$ siempre es mayor o a lo sumo igual que el número de filas ${ }^{+} a \mathrm{y}^{+} c$. Dos casos extremos donde resulta fácil comprobar la validez de la Ec. (5) es cuando todas las filas contienen signos $+($ obteniéndose $14 \geqslant 7)$ o cuando todas contienen signos - (obteniéndose $0 \geqslant 0)$.

Como las predicciones cuánticas son probabilísticas, para que la Ec. (5) pueda ser contrastada con

\begin{tabular}{|c|c|c|}
\hline$a$ & $b$ & $c$ \\
\hline+ & - & + \\
\hline- & + & + \\
\hline- & + & - \\
\hline+ & - & + \\
\hline+ & + & - \\
\hline- & + & + \\
\hline+ & + & - \\
\hline
\end{tabular}

Figura 4: Cada columna de la tabla representa un eje determinado, y cada fila representa una medida de spin sobre dicho eje. Sin importar como se complete la tabla, siempre se cumple la desigualdad WD. la MC es necesario convertirla en una desigualdad entre probabilidades conjuntas. Denotemos como $N$ al número total de mediciones conjuntas para cada par de partículas 1 . Como $N>0$, si ambos miembros de Ec. (5) se dividen por Nla desigualdad se mantiene:

$$
\frac{n u m\left({ }^{+} a, b^{+}\right)}{N} \leqslant \frac{n u m\left({ }^{+} a, c^{+}\right)}{N}+\frac{n u m\left({ }^{+} c, b^{+}\right)}{N}
$$

Si se supone que $N$ es grande y que los pares de protones han sido emitidos de forma aleatoria desde la fuente, entonces cada término de la Ec. (6) corresponde a la definición elemental de probabilidades conjuntas, vale decir, cada término expresa una simple proporción, definida como el cociente entre el número de eventos de interés y el número de eventos totales:

$$
\operatorname{prob}\left({ }^{+} a, b^{+}\right) \leqslant \operatorname{prob}\left({ }^{+} a, c^{+}\right)+\operatorname{prob}\left({ }^{+} c, b^{+}\right)
$$

La Ec. (8) establece que si se admiten las reglas del argumento EPRB, entonces la proporción de medidas conjuntas de spin con componentes ${ }^{+} a \mathrm{y} c^{+}$, sumada a la proporción con componentes ${ }^{+} c y b^{+}$, es siempre mayor o igual que la proporción con componentes ${ }^{+} a$ y $b^{+}$. En la siguiente sección se contrasta la Ec. (8) con las predicciones estadísticas de la MC.

\section{4. ¿Es posible eludir las reglas de la mecánica cuántica?}

Con el propósito de contrastar la desigualdad WD con las predicciones estadísticas de la MC, conviene reescribir la Ec. (7) del siguiente modo:

$$
\operatorname{prob}\left({ }^{+} a, c^{+}\right)+\operatorname{prob}\left({ }^{+} c, b^{+}\right)-\operatorname{prob}\left({ }^{+} a, b^{+}\right) \geqslant 0
$$

A partir de reglas elementales de la MC se puede demostrar que cada una de las proporciones o

\footnotetext{
${ }^{1}$ Dado que existen tres ejes, $a, b$ y $c$, el número de pares ordenados de ejes distintos es $3 \times 2 \times 1=6$. Además, cada uno de estos pares ordenados puede generar cuatro combinaciones para los valores $(+)$ y $(-)$, de modo que existe un total de $6 \times 4$ $=24$ conjuntos de pares de mediciones sobre ejes distintos de componentes de spin. Si denotamos como $n_{1}, n_{2}, n_{3} \ldots, n_{24}$ al número de mediciones conjuntas para cada par, se sigue que $N=n_{1}+n_{2}+n_{3}+\ldots+n_{24}$.
} 
probabilidades conjuntas de la Ec. (8) vienen dadas por las siguientes expresiones $[25,26]$ :

$$
\begin{aligned}
& \operatorname{prob}\left({ }^{+} a, c^{+}\right)=\frac{1}{2} \sin ^{2}\left(\frac{\theta_{a}-\theta_{c}}{2}\right) \\
& \operatorname{prob}\left({ }^{+} c, b^{+}\right)=\frac{1}{2} \sin ^{2}\left(\frac{\theta_{c}-\theta_{b}}{2}\right) \\
& \operatorname{prob}\left({ }^{+} a, b^{+}\right)=\frac{1}{2} \sin ^{2}\left(\frac{\theta_{a}-\theta_{b}}{2}\right)
\end{aligned}
$$

donde $\theta_{a}, \theta_{b}$ y $\theta_{c}$ corresponden a los ángulos que forman los ejes $a, b$ y $c$ respecto de un eje de referencia arbitrario. Las tres ecuaciones anteriores representan las predicciones de la MC para las proporciones de medidas conjuntas establecidas por la Ec. (8). A simple vista no es posible determinar si la MC predice resultados experimentales distintos de los que predice el argumento EPRB. Para verificar esto último, es necesario reemplazar las Ecs. (9), (10) y (11) en la Ec. (8):

$\sin ^{2}\left(\frac{\theta_{a}-\theta_{c}}{2}\right)+\sin ^{2}\left(\frac{\theta_{c}-\theta_{b}}{2}\right)-\sin ^{2}\left(\frac{\theta_{a}-\theta_{b}}{2}\right) \geqslant 0$

Como es fácil comprobar, si se escogen los ángulos de modo que $\theta_{a}-\theta_{c}=\theta_{c}-\theta_{b}=90^{\circ}$ y $\theta_{a}-\theta_{b}=180^{\circ}$ (el eje $c$ es bisectriz del ángulo $\theta_{a}-\theta_{b}$ ), no se genera conflicto con la Ec. (12) (ver Fig. 5). En efecto, al introducir estos valores en la Ec. (12) se obtiene:

$$
\sin ^{2}\left(45^{\circ}\right)+\sin ^{2}\left(45^{\circ}\right)-\sin ^{2}\left(90^{\circ}\right) \geqslant 0
$$

Evaluando cada término en la Ec. (13) resulta $0 \geqslant$ 0 , lo que siempre es verdadero. Esta es, básicamente, la situación considerada por Einstein, Podolski, Rosen y Bohm, quienes limitaron la discusión al caso en que los ejes de medición están orientados de forma paralela o perpendicular. Por lo tanto, tal

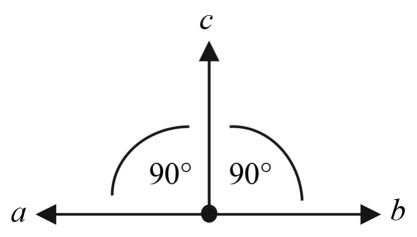

Figura 5: Orientaciones relativas de los ejes $a, b$ y $c$ para las medidas de spin bajo las reglas del argumento EPRB, donde se cumple que $\theta_{a}-\theta_{c}=\theta_{c}-\theta_{b}=90^{\circ}$ y $\theta_{a}-\theta_{b}=$ $180^{\circ}$. como descubrió Bell, en el contexto del argumento EPRB no puede surgir ningún conflicto con las predicciones estadísticas de la MC.

Considérese ahora una situación más general, donde las orientaciones relativas de los ejes $a, b$ y $c$ son arbitrarias (ver Fig. 6). Escojamos entonces los ángulos de modo que $\theta_{a}-\theta_{c}=\theta_{c}-\theta_{b}=\theta$ y $\theta_{a}-\theta_{b}=$ $2 \theta$ (nuevamente se supone que el eje $c$ es bisectriz del ángulo $\theta_{a}-\theta_{b}$ ). En estas condiciones, la Ec. (12) puede escribirse como:

$$
2 \sin ^{2} \frac{\theta}{2}-\sin ^{2} \theta \geqslant 0
$$

Si en la Ec. (14) se toma $\theta=45^{\circ}$ se obtiene $0 \geqslant 0,208$ de modo que la desigualdad es violada. En general, la Ec. (14) será violada para todo ángulo tal que $0<\theta<90^{\circ}$ o $270^{\circ}<\theta<360^{\circ}$. Para observar la violación de la desigualdad WD gráficamente, a partir de la Ec. (14) conviene definir la siguiente función:

$$
f(\theta)=2 \sin ^{2} \frac{\theta}{2}-\sin ^{2} \theta
$$

Naturalmente, cualquier valor de $f(\theta)$ menor que cero implica una violación de la desigualdad WD. Al graficar la Ec. (15) poniendo $f(\theta)$ en el eje de las ordenadas y $\theta$ (en grados) en el eje de las abscisas, se observa claramente que la curva se encuentra bajo el eje $f(\theta)=0$ para todos los ángulos tales que $0<\theta<90^{\circ}$ o $270^{\circ}<\theta<360^{\circ}$ (ver Fig. 7).

Por lo tanto, la desigualdad WD proporciona un test que permite responder la pregunta que encabeza esta sección: ¿Es posible eludir las reglas de la MC? Como se ha mencionado en la introducción, los resultados obtenidos hasta ahora confirman las predicciones cuánticas, revelando una clara violación de la desigualdad de Bell [7-10]. Así, la evidencia empírica sugiere que no es posible eludir la reglas

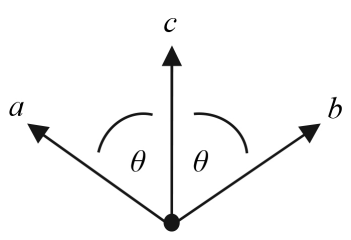

Figura 6: Orientaciones relativas de los ejes $a, b$ y $c$ para las medidas de spin bajo las condiciones más generales impuestas por la desigualdad de Bell. Para ilustrar las relaciones entre los ángulos, un caso particular de interés se obtiene cuando $\theta_{a}-\theta_{c}=\theta_{c}-\theta_{b}=45^{\circ}$ y $\theta_{a}-\theta_{b}=2 \times 45^{\circ}$ $=90^{\circ}$. 


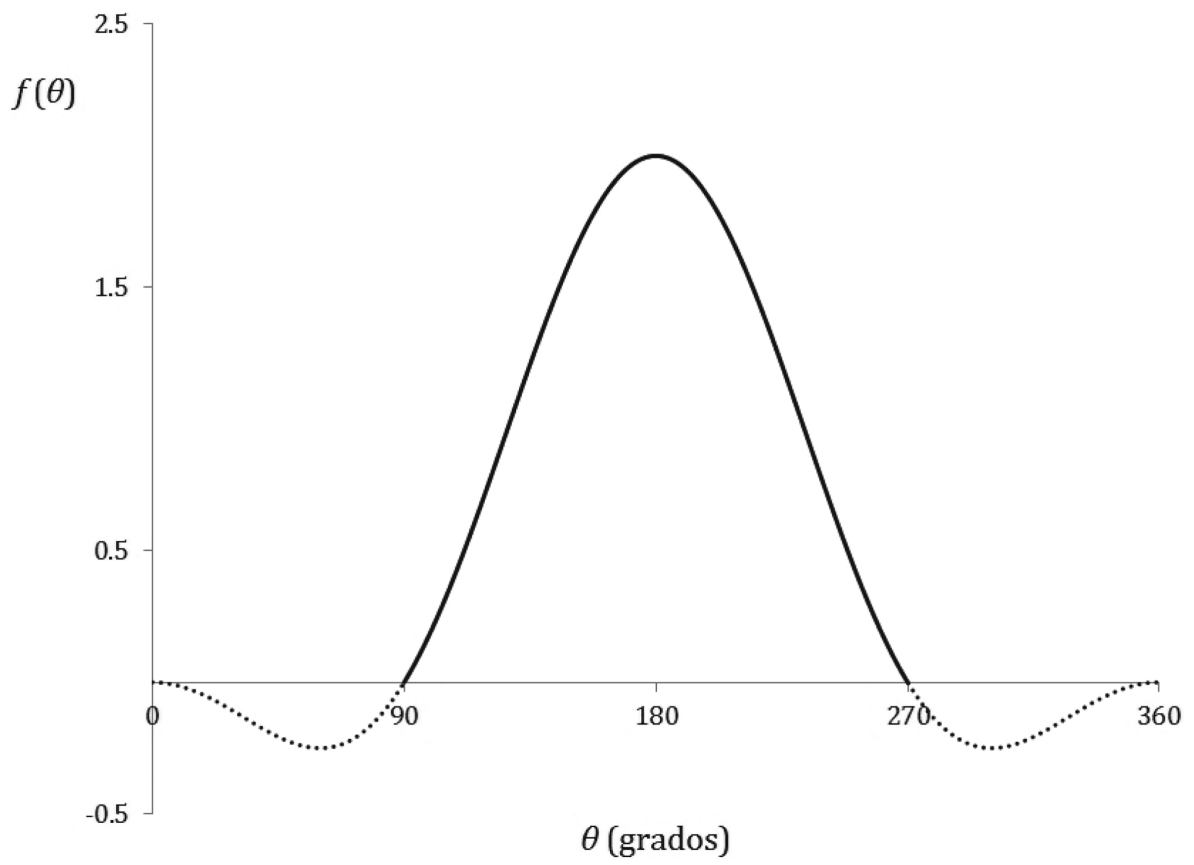

Figura 7: La desigualdad de Bell es violada cuando la curva se encuentra bajo el eje $f(\theta)=0$, lo que sucede para todo ángulo $\theta$ tal que $0<\theta<90^{\circ}$ o $270^{\circ}<\theta<360^{\circ}$.

de la MC en el marco de un modelo tipo EPR. Sin embargo, aun queda por responder otra pregunta fundamental: ¿Cuál es el significado físico de la violación de la desigualdad de Bell? Según ha sostenido el propio Bell, la dificultad de fondo parece relacionarse con el principio de localidad de Einstein $[5,6]$, lo que sugiere fuertemente que dos objetos separados una distancia arbitrariamente grande pueden interactuar físicamente mediante influencias que se propagan más rápido que la luz. Sin embargo, dado que el argumento EPRB también depende del criterio de realidad (ver sección 2), y dado que algunos autores, especialmente Bohr y sus seguidores, han cuestionado la aplicabilidad del concepto de realidad física en el marco de la MC, también es posible que la violación del teorema de Bell se deba a que el realismo no es válido a nivel cuántico. Más aun, y llevando la lógica del argumento al extremo, la violación de la desigualdad de Bell bien podría ser consecuencia de que tanto la localidad como el realismo no son válidos. No obstante, dado que para explicar la violación de la desigualdad de Bell basta con renunciar a la localidad, rechazar tanto ésta como el realismo parece ser una opción que Bell considera demasiado radical y costosa.

En cualquier caso, frente a este panorama cabe preguntarse si la violación de la desigualdad de Bell implica que Bohr derrotó a Einstein. Como ha señalado Bell, sería equivocado afirmar que la victoria final pertenece a los oponentes de Einstein, pues éstos no conocían el análisis desarrollado antes [6]. Sin embargo, lo que parece claro es que la creencia de Einstein en un universo gobernado por la localidad y el realismo es falsa, porque Bell ha probado que el argumento EPRB es incompatible con las predicciones estadísticas de la MC.

\section{Agradecimientos}

Quisiéramos agradecer a Michael Van Sint Jan y Daniela Balieiro por sus valiosos comentarios durante la elaboración de este artículo.

\section{Referencias}

[1] A. Einstein, B. Podolsky and N. Rosen, Physical Review 47, 777 (1935).

[2] D. Bohm, Quantum Theory (Dover Publications Inc, New Jersey, 1989).

[3] B. d'Espagnat, Scientific American november, 158 (1979).

[4] N. Bohr, Physical Review 48, 696 (1935).

[5] J.S. Bell, Physics 1, 195 (1964).

[6] J.S. Bell, Le Journal de Physique Colloques 42, 41 (1981).

[7] A. Aspect, J. Dalibard and G. Roger, Physical Review Letters 49, 1804 (1982). 
[8] W. Tittel, J. Brendel, H. Zbinden and N. Gisin, Physical Review Letters 81, 3563 (1998).

[9] G. Weihs, T. Jennewein, C. Simon, H. Weinfurter and A. Zeilinger, Physical Review Letters 81, 5039 (1998).

[10] D. Salart, A. Baas, J.A.W. van Houwelingen, N. Gisin and H. Zbinden, Physical Review Letters 100, 1 (2008).

[11] H.P. Stapp, Nuovo Cimento 29B, 270 (1975).

[12] E.P. Wigner, American Journal of Physics 38, 1005 (1970).

[13] G. Blaylock, American Journal of Physics 78, 111 (2010).

[14] T. Maudlin, American Journal of Physics 78, 121 (2010).

[15] R.B. Griffiths, American Journal of Physics 79, 954 (2011).

[16] M. Nauenberg, American Journal of Physics 83, 197 (2015).

[17] J. Bricmont, disponível em http://www.ijqf .org/ archives/1303 (2013).

[18] M.G. Alford, http://arxiv.org/pdf/1506. 02179v1.pdf (2015).

[19] M.D. Greenberger, M.A. Horne, A. Shimony and A. Zeilinger, American Journal of Physics 58, 1131 (1990).

[20] F. Kuttner and B. Rosenblum, The Physics Teacher 48, 124 (2010).

[21] P.G. Kwiat and L. Hardy, American Journal of Physics 68, 33 (2000).

[22] N.D. Mermin, American Journal of Physics 58, 731 (1990).

[23] K. Jacobs and H.M. Wiseman, American Journal of Physics 73, 932 (2005).

[24] L. Maccone, American Journal of Physics 81, 854 (2013).

[25] J.J. Sakurai, Modern Quantum Mechanics (Addison Wesley, New York, 1994), p. 229-232.

[26] L. de la Peña, Introducción a la Mecánica Cuántica (Fondo de Cultura Económica, México, D.F., 2006), $3^{\mathrm{a}}$ ed., p. 540-549. 